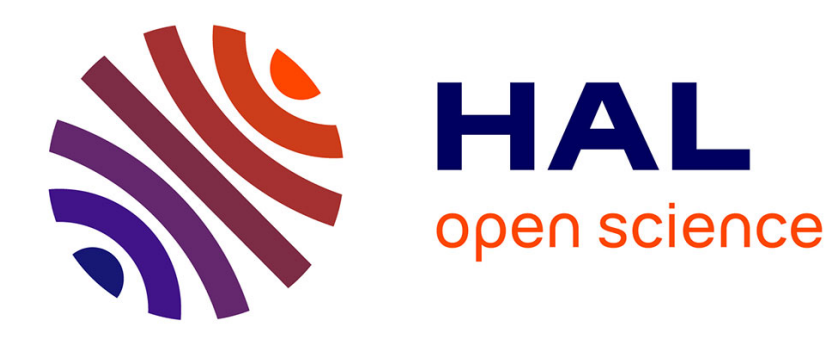

\title{
Dosage du radium par la mesure de l'émanation dégagée Pierre Curie
}

\section{To cite this version:}

Pierre Curie. Dosage du radium par la mesure de l'émanation dégagée. Radium (Paris), 1910, 7 (3), pp.65-70. 10.1051/radium:019100070306500 . jpa-00242397

\section{HAL Id: jpa-00242397 https://hal.science/jpa-00242397}

Submitted on 1 Jan 1910

HAL is a multi-disciplinary open access archive for the deposit and dissemination of scientific research documents, whether they are published or not. The documents may come from teaching and research institutions in France or abroad, or from public or private research centers.
L'archive ouverte pluridisciplinaire HAL, est destinée au dépôt et à la diffusion de documents scientifiques de niveau recherche, publiés ou non, émanant des établissements d'enseignement et de recherche français ou étrangers, des laboratoires publics ou privés. 


\title{
MÉMOIRES ORIGINAUX
}

\author{
Dosage du radium par la mesure de l'émanation dégagée \\ Par Mme P. CURIE \\ [Faculté des Sciences de l'aris.]
}

Le dosage du radium par l'émanation yu il dégage en un temps donné a été proposé de divers côtés et a été effectivement utilisé dans différents laboratoires pour des travaux dont certains ont une arande importance. La méthode qui a été employéc le plusouvent est celle qui consiste à chasser par l'ébullition l'émanation accumulée pendant un temps connu dans un vase clos contenant une solution de radium, et à transporter cetteémanation dans un appareildemesur.es.

J'ai tout spécialement étudié une méthode de dosage du radium par l'émanation qu'il dégage. Cette méthode, employée couramment au Jaboratoire de radioactivité de laris depuis plusieurs annces, s'est montrée d'un emploi très pratique el domne des résullats d'une très grande précision. C'est pourquoi elle sera décrite ici arec quelques détails.

La méthode consiste à entrainer par un courant d'air à froid l'émanation dégagée par une petite quantité (quelques $\mathrm{cm}^{5}$ ) d'une dissolution contenant du radium.

Les deux formes d'appareils en verre que j'emploie pour y placer la dissolution sont représentées dans la figure 1 . On les nomme barboteurs. Le barboteur a

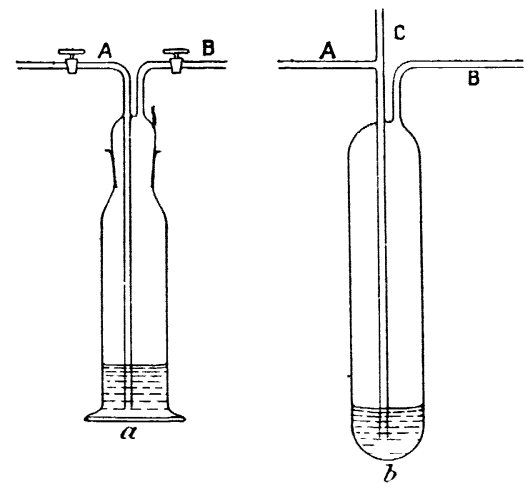

Fir. 1 .

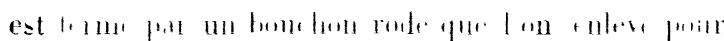

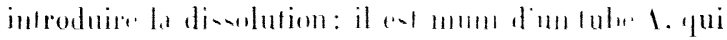

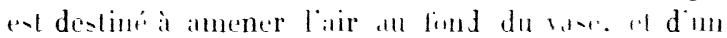
tube de degagement B: ces deur mbev-unt à robli-

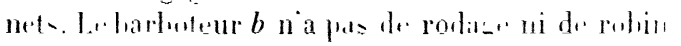

1. 7 . la solution est introduite par la tubulure $\mathrm{C}$ au mosen d'une pipette à pointe longue et fine; aprìs introduction de la dissolution la tubulure c est fermée à la lampe. Le tube I sert pour l'arricée de liar. el le tule $B$ est un tube de diogagement; ces deux tubes sont élirés en pointe line, et ils restent fermés à la lampe pendant que l'émanation śaccumule. de sorte que l'appareil est rigoureusement clos el qu'ancune fuite d'émanation ne peut aroir lieu. Le harrotenr a n'offre pas la même sécurité puisque sil fermeture comporte un rodage et deux robinets; il est toutefoiv d'in emploi plus pratique et sert pour l'usage rourant, tandis que le barboteur $b$ est employé pour des recherches de précision. Le volume total des barlonteurs est, en général, de $25 \mathrm{~cm}^{3}$ : mais on peut (111ployer des barboteurs plus petits ou plus arands.

On introduit dans le barboteur un petit volume de la solution proposée, de manière que le tube A plonge dans la dissolution sur quelques millimitres de longueur.

L'appareil qui sert pour mesurer l'activité de l'émanation produite par la solution est un condensateur à gaz du modèle représenté dans la ligure 2 . Le condensateur est muni de deux robinets: on doit rérifier qü̈l est parfaitement étanche. Le transport de l'émanation contenue dans un barboteur de la form. $a$ dans le condensaleur de mesures se fait de la manière suisante: la tubulure B du barbotenr an mi-e col communication au mojen d'im bon tube de cionut-

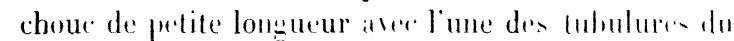
comden-atene din-lenpel on a fait prealablement l. vide; le robinet I itant lermé. on etallit lat commmunication entre le barboteur el le rondensilenr: lair

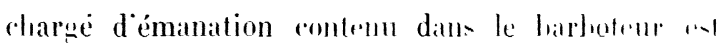

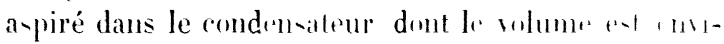

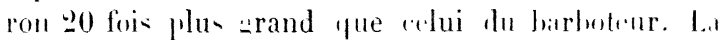

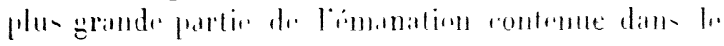

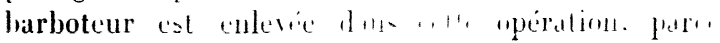

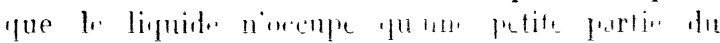

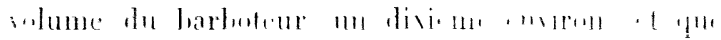

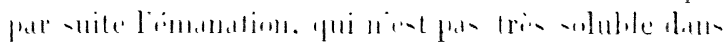

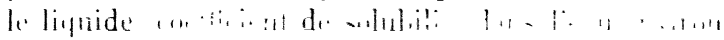

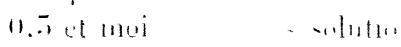

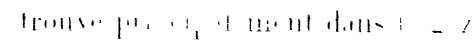


In ferme alors la communicalion arec le condensiteur : on nurre peu à peu le robinet $\Lambda$ et on laisse rentrer dans le barboteur de lair inactif qui, barbo-

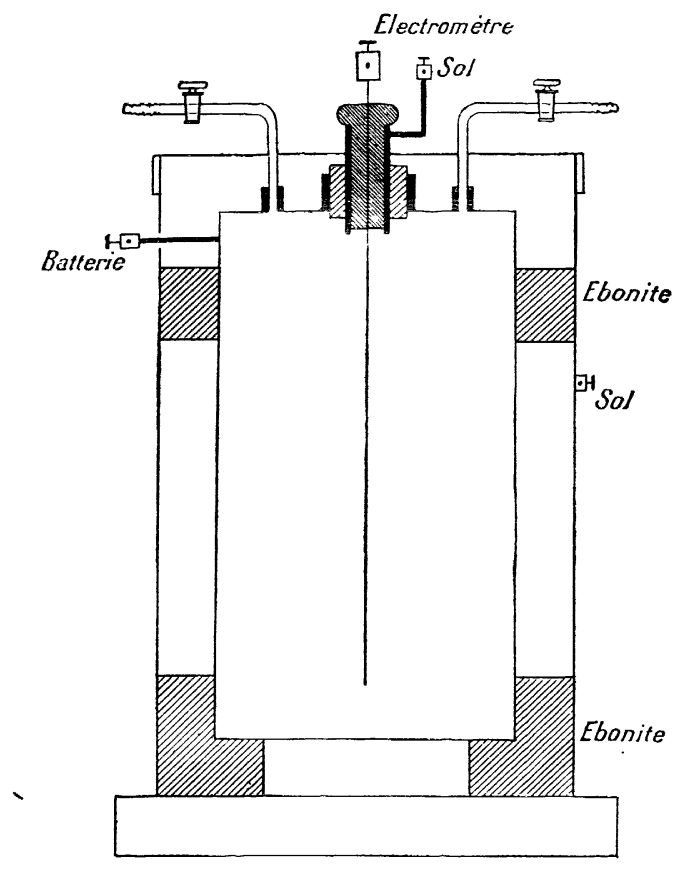

Fig. 2.

tant dans la solution, cutraine l'émanation yui y est contenue. (Guand la pression atmosphérique est rélablic dans le barboteur, on lait une nouvelle aspiration en ouvrant le robinet du còté du condensateur pendant que le robinet A est fermé.

Je me suis assurée que par une succession d'aspirations et de rentrées d'air on peut pratiquement enlever la totalité de l'émanation contenue dans le barboteur pour des temps d'accumulation supéricurs à quelques heures. Il est nécessaire d'opérer toujours de la mème manière; jai obtenu d'excellents résultats en produisant trois aspirations suivies de trois rentrées d'air; aprìs quoi, le robinel A étant ouvert, on laisse rentrer dans le condensateur un courant d'air traversant le barboteur sous pression atmosphérique et ayant toujours à peu prè la mimo vitesse régléc par une manu'ure du robinet du condensateur. Ilans mes expériences l'ensemble des trois aspirations et des trois rentrées dair demande is minules: le courant daair régulier passe ensuite pendant 10 minutes. II est tris facile de shabituer à eflectuer toujours les opérations de la mème manière et à apprécier très exactement la vitesie du courant dair par laspect du barbotage. Le liquide se troure alors upre- chayue opération dans un cit.t parfiritement défini et ne garile quine quantité dentranation négligeable par rapport i coll" qui se forme en quelques heures. Les quantiles d'émanation extraites à des intervalles de temps igaux sont alors très exactement égales pour des temps d'accumulation supérieurs à quatre heures.

Lair que l'on introduit dans les barboteurs est pris au dehor au moyen d'un tuyau de plomb, filtré au travers de coton de verre et lavé dans un flacon à eau distilléc inactive.

Quand on emploie un harboteur de forme b, l'ouverture des tubulures se fait en cassant leurs pointes dans les tubes de caoutchonc qui les relient au condensateur et au flacon laveur; les robinets yui servent pour la manneurre sont alors celui du condensateur et celui du flacon laveur. Iprès l'opération on ferme à nouveau les pointes des tubulures à la lampe.

Quand l'opération est finie, la pression atmosphérique n'est pas encore rétạblie dans le condensateur. On laisse celui-ci sous pression réduite, et l'on attend que par suite du développement de la radioactivité induite le courant ait atteint la valeur maximum, qui peut facilement être mesurée arec une grande cractitude. Il y a arantage à rétablir la pression atmosphérique dans le condensateur seulement quelques minutes arant la mesure parce que, si l'appareil n'était pas parfaitement ćtanche, la petite perte d'émanation yui pourrait en résulter se trouverait diminuće. On peut faire une mesure du courant de saturation entre $\bar{j}$ heures et demic et 4 heures à partir de l'aspiration de l'émanation. Il est bon de faire 2 séries de mesures à une demi-heure d'intervalle.

Le courant est généralement mesuré par li méthode du quartz piézoélectrique, mais on peut aussi utiliser un dispositif dıfférent. La valeur du courant de saturation pour une même quantité d'émanation dépend des dimensions du condensateur et de la densité du gaz qui ! est contenu. Ces conditions influent ('n effet sur la maniìre dont se trouvent utilisés les rayons émis par l'émanation et la radioactivité induite. Plus toutes les dimensions du condensateur sont grandes et plus la densité du gaz est élevée, plus le courant est intense, à quantité d émanation égale. Les dimensions des condensateurs employes étaient toujours les mèmes, il était donc seulement nécessaire de préciser linfluence de la densité du gaz et de ramener toutes les mesures à une densité considérée comme normale. La correction à appliquer à cet 'ffet a été détermince par l'expérience. Ine certaine quantité démanation se trouvant dans un condensateur relié à un manomètre, et le courant avant atteint sa valeur maximum, on faisait la mesure du courant en augmentant par degrés la quantité d'air contenuc dans le condensateur, de manière à aroir la valeur du courant pour une série de pressions comprenant la pression atmosphérique et s'en écarlant dans les deux s'm- de la valeur de quelques centimètres de mercure. lia courbe epérimentale ainsi obtenue a permis d'établir la correction due à la variation de pression de lair atmosphérique: la correction due à la varia- 
tion de la température ambiante a été déduite en admettant que la temperature no intervient que pour modifier la densiti de l'air.

Arec les dimension- des condenateur-emplosés la formule de correction est lit suirante:

$$
\Sigma=i 0.010117(\bar{Z}(60)-1)+1) .002(t-1)
$$

E représentant la correction à ajouter au courant mesuré $i$ pour le ramener à la valeur qu'il aurait eue si lair du condensateur arait été à la pression de $760 \mathrm{~mm}$. de mercure el i la température de 15 ", alors que la pression et la température de l'air an moment du remplissage final ont élé effectivement $/ 1 \mathrm{~mm}$. de mercure el $t$ degrés. La correction peut alleindre Q pour [1)(1 de la valeur mesurée, el son emploi est utile.

Le condensateur qui a serri pour une mesure est ensuite purgé de l'émanation qu'il contient; pour cela on ! fait le vide el on laisse ensuite rentrer de l'air inactif séchè et filtré. Quand cette opération a été répétée plusicurs fois, le conclensateur est ahandonné pendant une journéc pour permettre l'extinclion de la radioactivité induite. 11 peut après cela resservir pour une nouvelle mesure. Toutefois il peut conserver une pelite activité que l'on doit faire intervenir comme correction aux mesures et qui doit ètre aussi faible que possible. Pour protéger le condensateur contre les accidents, on interpose entre le barboteur et le condensateur un très petit tube contenant un pelı de coton de verre. On peut avoir un certain nombre de condensateurs; s'ils ont les mèmes dimensions, ils sont parfaitement interchangeables ainsi que je m'en suis assurée fréquemment.

L'air chargé d'émanation qui pénètre dans le condensateur est très humide, linterposition de tubes desséchants peut occasionuer des erreurs telles que l'absorption de petites quantités d'émanation par ces matières. Cette interposition peut ètre évitée si l’on a soin de se servir d'amb,re pour les pièces isolantes du condensateur: l'ambre conserve en effet dans l'air humide ses propriétés isolantes à un degré sulfisant, pour que la mesure puisse se faire très exactement par une méthode de zéro ; l'ébonite ne peut pas rendre le mème service. Apris chaque opération il est bon de dessécher le condensateur.

J:ai effectué un grand nombre d'expériences pour préciser les conditions qui permettent dobtenir des rivultils concordints.

La fuanliti d'iemanation q acemmulec pendant le temps $t$ e- domnce par lit formule.

$$
\eta=-\Delta p r\left(1-e^{-\frac{t}{t}}\right)
$$

où 9 désinge la vie mosenne de lémanations, l" l" fwill de radium préent et $\lrcorner l$. lebit d'imanation fur - ramme de radium en unites arbitraires.

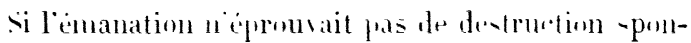

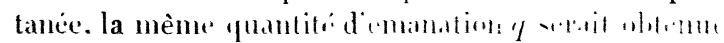

(n) un temps $t$, que je nomme temps reduit: on at.

$$
\begin{gathered}
q=\Delta, t_{1} \\
\text { par suite } \quad t_{r}=s\left(\begin{array}{ll}
1 & e-\frac{1}{r_{1}}
\end{array}\right)
\end{gathered}
$$

Le tempre reduit augmente arec $/$ et tond ror la valeur o quand $t$ tend vers linfini. Pratiquement, la limite est atteinte arec une prande approsimation pour $t=\mathbf{5 0} 0$ jours.

La quantite $\frac{\eta}{l}=\Delta \rho$ deit etre more comstante pune un barboteur détermine contenant un poids lise p de radium. Soit Ri la valeur de ce rapport. Pour derr barboteurs contenant des poids différents $f$ el $/ 1$ de

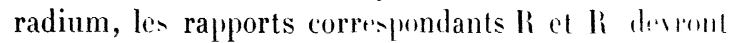
vérifier la relation

$$
\frac{\mathrm{R}^{\prime}}{\mathrm{R}}=\frac{p^{\prime}}{p}
$$

Il sulfira done de commaitre la valeur du rapprorl li pour une solution contenant une quantite comme de radium pour pouvoir doser par comparaison le radium contenu dans toute autre solution. Pour prouver ylue l'emploi de la méthode est légitime, il est nécessair. de s'assurer que le rapport R est indépendant du temps d'accumulation et yu'il est proportionnel ì la teneur de la solution en radium.

Si la constante de temps $\theta$ de l'émanation e- connue, le temps réduit $t_{r}$ peut facilement ètre calculé pour une valeur quelconque du temps d'accumulation $t$. On peul, en partitulier, construire une table qui fournit les valeurs de $t_{r}$ correspondant à des valeurs de $t$ croissantes par intervalles convenables (d'heure en heure jusqu à 18 heures, ensuite par quart de jour, par demi-jour et finalement par jour jusqu à 70 jours). Il est aussi utile d’indiquer dans la table les valeurs des fonctions $e^{-\frac{t}{\sigma}}$ et $1-e^{-\frac{t}{j}}$ qui se trouvent calcukes en mème tempr. Cne table de ce genre est depuis plusieur anne'es d'usage comrant au laboratoire Curio. Lat have adoptée primitiremenl

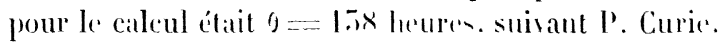
1 la suite deypériences plus récentes'. on pent in!mettre pue lat véritable valeurde g est très woisime de las.2 heures. Ciest ce nombre yui a éte adopti comme base pour la construction d'une table. yui a été publiée antérieurement par II. holowrat

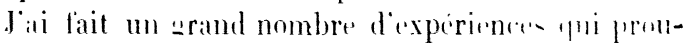

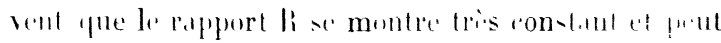

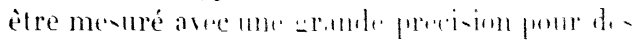

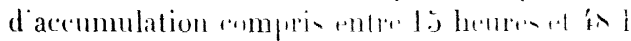

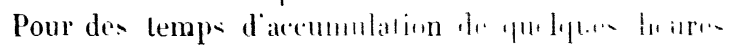

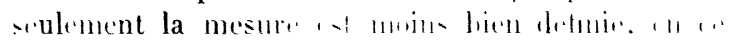

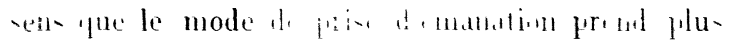

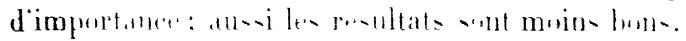

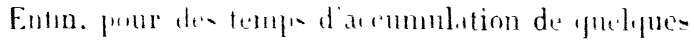

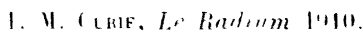

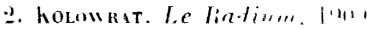


jour: ct davantage, $R$ conserve approximativement la mème valeur que pour un jour: loulefois, les mesures sont beaucoup moins concordantes, et la valeur Irouréc pour R est le plus sourent trop faihle. l'écart pouvant atleindre ì pour 100 et mème davantage.

Guand on emploie des barboteurs à robinets, toute valeur de $k$ trop faible peut être attribuce à un défaut de fermeture, mais arec des barboteurs entièrement clos cette cause d'erreur est supprimée. Il est possible que pour des temps daccumulation longs il puisse se produire une occlusion partielle de l'émanation dans le verre. On peut aussi remarquer que si l'on obtenait toujours pour' $t=\bar{j} 0$ jours une valeur de $\frac{q}{t_{r}}$ constante et inférieure à celle que l'on obstient pour $t==1$ jour, ce lait serait me indication pour penser que la valeur admise pour $\theta$ est trop forte; en effet, si par exemple on modifie de ò pour 100 la valeur, de $\theta$ le temps réduit qui correspond à $t=\infty$ est modifié dans la mème proportion, tandis que les temps réduits qui correspondent aux temps daccumulation inférieurs à deux jours éprouvent une ríduction inféricure à 1 pour 100 . Les résultats obtenus avec des valeurs de $t$ comprises entre 5 et 50 jours ne sont pas assez réguliers pour que l'on puisse s'en servir dans le but de fixer la valeur de la constante $\%$.

Voici, à litre d'exemple, quelques-unes des nomhreuses sceries de mesures effectuées; on a indiqué fir quelques barboteurs la valeur de li pour divers lomps d'accumulation $t$.

\begin{tabular}{|c|c|c|c|}
\hline \multicolumn{2}{|c|}{ Ba boteur is rolinels } & \multicolumn{2}{|c|}{ Barboteur sans robinets } \\
\hline I heure- & R & I heures & $\mathrm{R}$ \\
\hline $\begin{array}{c}30.17 \\
121,15 \\
51,41 \\
67,4 \\
68,9 \\
24,0 \times \\
792 \\
12.12\end{array}$ & $\begin{array}{l}2,15 \\
2.10 \\
2,15 \\
2,15 \\
2,13 \\
2,13 \\
1,97 \\
2,14\end{array}$ & $\begin{array}{c}16.8 \\
26,42 \\
21,5 \\
21,17 \\
26,5 \\
6,27 \\
17.5 .7 \\
2.42 \\
21,68 \\
0,908\end{array}$ & $\begin{array}{l}14.74 \\
14.18 \\
14,27 \\
14,28 \\
14.29 \\
14.58 \\
14,17 \\
14,47 \\
14.07 \\
14.58\end{array}$ \\
\hline
\end{tabular}

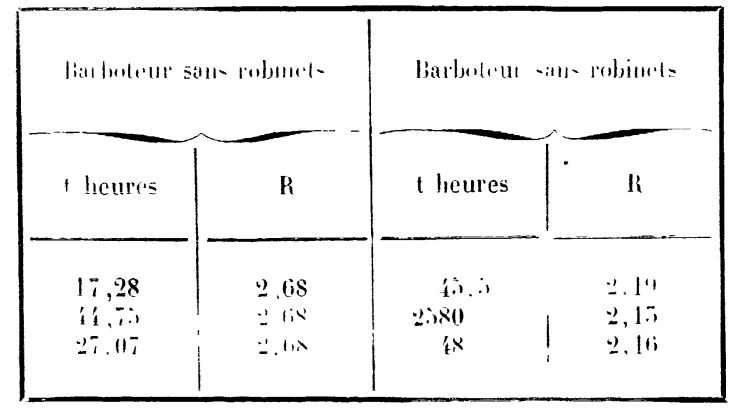

En emplosant un temps d'accumulation entre un et deux jours et en ayant soin de se servir de barboteurs tris propress et d'effectuer la prise d'émanation très régulièrement, on obticnt unc précision de $0, \vdots$ pour 100. Licreur à craindre sur la constante "s ne peut influencer les résultats d'une manière appríciable si $t<48$ heures.

Si l'on a obtenu une bonne concordance pour la valeur de $R$ mesurée arec un même barboteur, on peut constater que les valeurs de $R$ pour deux barboteurs différents sont très exactement proportionnelles aux poids de radium contenus dans le barboteur à l'état de dissolution. On peut alors préparer une solution étalon arec un sel de radium pur et déterminer en valeur absolue l'intensité du courant do saturation que l'émanation fournie par cette solution par unité de templs, peut produire dans un condensateur de forme déterminée.

Cet étalonnage sert ensuite de base pour tous les dosages que l'on roudra effectuer; il constitue une opération délicale yui doit ètre faite avec le plus grand soin en raison de son importance. Voici comment cette opération a été exécutée.

Le sel de radium que j’ai utilisé était le chlorure de radium parfaitement pur que javais préparé en 1907 et qui marail servi pour la détermination du poids atomique du radium; le poids atomique oblenu élait égal à 226 , ò.

Le sel yui renait de cristalliser d'une solution contenant de l'acide cỏlorhydrique a été séché à l'éture et privé de son eau de cristallisation. Ensuite quelques fragments conservant la forme de cristaux ont été choisis, placés dans un creuset de platine et pesés après dessiccation arec les mêmes précautions que celles qui ctaicut habituelles pour une détermination de poids atomique, avec la mème balance précise 't rapide. Le poids de chlorure de radium anhydre utilisé élait de 0,02 à 0,05 gramme.

Le sel a été introduit dans un flacon de verre à bouchon rodé parfaitement propre. Ce flacon avait une contenance d'environ $200 \mathrm{~cm}^{\bar{j}}$; il était pesé ì un milligramme près. En versant sur le sel un peu d'eau qui avait été redistillée dans un appareil en platine, on a obtenu la dissolution du sel sans aucun résidu. Le volume de la solution a alors été amené à $150 \mathrm{~cm}^{5}$ environ el une goutte dacide chlorhydrique trís pur a été ajoutée. Le poids tolal de la dissolu lion élait déterminéc par une pesée précise au milligramme.

La quantité de chlorure de radium pur qui élait utilisée pour une mesure de l'activité de l'émanation dégagéc est de l'ordre de $10^{-6} \mathrm{gr}$. Il était don néceso - ire de faire des barboteurs avec une solution beauroup plus diluce que la solution principale. Pour cela on a fait sur celle-ci des prises de liquide destincés a la preparation de solutions plus diluén. Lat sulution 
principale avant étri fortement agitée dans le flacon fermi, on prend aree une pipette une quantité convenable $\left(1 \mathrm{~cm}^{5}\right.$ environ) de solution et on l'introduit dans un petit flacon de verre lin de ${ }^{\prime} \mathrm{cm}^{5}$ de rolum.. préalablement pesé arec soin sur une halance précis. au dixième de millį̧ramme. Le liquide étant introduit et le flacon louche. une nouvelle pesée fait connaitre le poids de la prise de solution. Celle-ci est alors introduite dans un flacon de $200 \mathrm{~cm}^{5}$ préalablement pesé et le volume du liquide est amené it $150 \mathrm{~cm}^{5}$ environ au moyen d'eau parfaitement pure. La pesée du flacon fait connaître alors le poids de la solution. Deux solutions 'auxiliaires ont été généralement préparées ainsi au moyen de la solution principale: pour chacune de ces solutions la quantité convenable pour un barboteur était de 0,5 gr. à $1 \mathrm{gr}$. Les solutions ayant été agitées, on prenait avec une pipette à pointe fine la quantité de liquide convenable et on l'introduisait dans un barboteur (fig. I, b) soigneusement pesé à la balance de précision; le poids du liquide utilisé était déterminé par une pesée, et l'on ajoutait un peu d'eau pure pour amener le volume à $2 \mathrm{~cm}^{\overline{3}}$ environ, puis on fermait le barbotrur. Plusieurs barboteurs ont généralement été faits avec chacune des solutions auxiliaires.

Les solutions sont repesces après chaque prise afin que l'on puisse se rendre compte si leur concentration varie par éraporation. Cetle variation est très lente et peut le plus souvent ètre négligée.

Les prises d'émanation ont été faites ainsi qu’il a ité décrit plus haut. Trois mesures étaient généralement faites pour chaque barboteur.

On mesurait pour un temps d'accumulation $t$ le rourant de saturation qui peut être obtenu dans un condensateur de forme déterminie 5 à $\mathbf{1}$ heures après l'introduction de l'émanation, c'est-it-dire quand le courant a atteint sa valeur maximum. L'intensité du courant était mesuréc au moỵen d'un quartz piézoélectrique en grammes par seconde; elle étail rappor- tée à une heure de temps réduit el ramenci à la juression normale et à une température de $15^{\circ}$. Les nombre ainsi obtenus sont inscrits dans la colonne $R$.

soit 1 le poids de sel contenu dans un barboteur. Le rapport $\frac{\mathrm{R}}{p}$ doit ètre constant. el sa connalissallu.t. suffit pour effectuer des dosages de radium, en conyriatul le mème condensateur à naz et la mime lame de quarls piézólectrique ou autre etalon de courant ins.rialile.

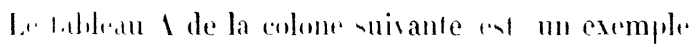
d'une determination de cerenre.

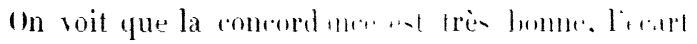
mavimum nitteignant pas $11, \therefore$ fwor lond.

prour obtenir un nombre asolu il fanl lade inter-

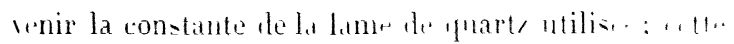

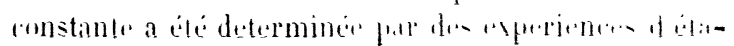

Tableau A.

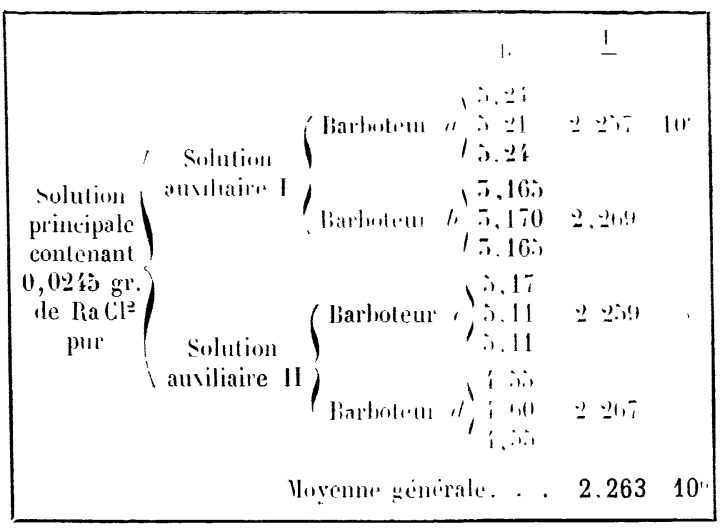

lonnage. La quantité délectriciti dégagée par la lame pour un poids connu est compraréc à celle que porte un condensateur à plateaux absolu pour une diflirem. de potentiel connue, fournie par une série d'eléments élalons '.

L'étalonnage a été effectué à l'aide d'un condensaleur de précision (plateaux de 20 centimètres do diamètre) et d'une batterie de 10 éléments Weston. Les lames utilisées donnent lieu ì un dégagement de 8 à 10 unités E.S. pour une traction d'un hilogramme.

Connaissant la constante du quartz on peut calculer l'intensité $i$ du courant maximum pour l'émanalion obtenue par gramme de sel el par heure de lemps réduil.

bien que les mesures soient, en général, très bonnes, on rencontre cependant d'assez grandes difficultés quand il s'agit d'obtenir la valeur absolue de $i$ arec une précision suffisante. Cés difficultén portent sur l'homogénéití des solutions et sur leur conservation. Arant chanfue prise ces solutions très diluées doivent ètre très fortement agitées, atin dr faire disparaitre les différencen do concentration locale. Les solutions doivent itre preparter arer le plus prind soin, en ' 'pui concerne la propreté des rases at la pureté des riactifs: res précaution ont pour but

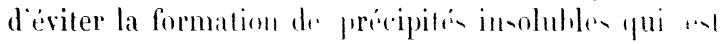

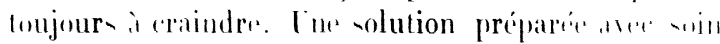

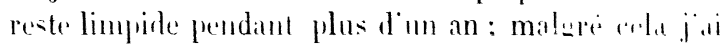

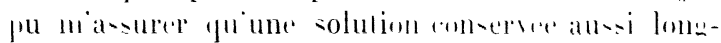

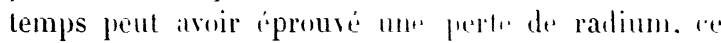
dernier étant probablement illowle prar le verre: la perte peut atteindre quelyue pour low en unt antex. Pour faciliter la comereration de solution-anciliair... il e-t ben de les additionner doune pelite fultutit. de

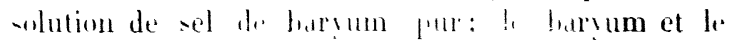
radium itant d. - he mate tres wi-ines et inmorphis. 111 jte

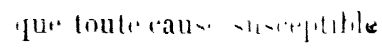

| 1) 111,... 1. H. 1 11\% 
d'iñolubiliser ces nuatières agira principalement sur lo haryum present en quantiti considérahlement plus grande que le radium.

Len résultals définitifs obtenus dans quelques sipie dieypipienes rientes conduisent it la valeur suirante de $i$

$i=2,00 \quad 10^{i}$ E.S. par aramme de Racl${ }^{2}$ et heure de lemps réduit.

$i=2.62$ 10. E.s. par gramme de Ra et heure de temps réduit.

Ces valeurs sont obtenues arec une précision d'environ 1 pour 100 , mais la dirergence arec une série de mesures antérieures $\epsilon$ st notablement plus grande (quelques pour 100). Cette divergence peut tenir à des imperfections des expériences. Mais on peut aussi se demander si la production d'émanation par une solution de radium n'est pas sujette à certaines varialions. Je telles variations pourraient avoir licu, s̈il evistait entre le radium et l'émanation un corps intermédiaire (Radium X), se séparant très difficilement du radium.

Il existe quelques indices en farcur de celte manière de voir. Le débit d'émanation dans des solutions préparées avec du chlorure de radium fraichement cristallisé a paru subir une légère augmentation. Dans d'autres solutions d'où l'on avait éliminé le radium par l'acide sulfurique, le débit d'émanation a progressivement diminué bien que la solution se soit conservée limpide.

(In peut déduire dı résultat numérique indiqué que la valeur du courant maximum pouvant itre obtenu avec l'émanation saturée d'un gramme de radium est égale à $2,6210^{\circ} \times 15 \overline{5}, 2$, soit $5,510^{5} \mathrm{E} . \mathrm{s}$. pour la chambre d’ionisation utilisée.

Ce nombre est obtemu avec les conditions expérimentales suivantes :
Le conden-ateur de mesures est un vase cylindrique dont roici lie dimensions :

$$
\begin{array}{ll}
\text { Ilauteur } & 12, \overline{\mathrm{cm}} ; \\
\text { l)iamc̀tre intéricur } & 6, \overline{\mathrm{cm}} \text {; } \\
\text { Volume } & 4 \hat{4} \mathrm{~cm}^{-5} \text { environ. }
\end{array}
$$

L'électrode centrale est une tige de $\overline{5} \mathrm{~mm}$. de diamètre qui arrive à la distance de $1 \mathrm{~cm}$. du fond.

Le courant de saluration a toujours été atteint; la diffórence de potentiel entre les armatures du condensateur était de 800 volts.

On peut d'ailleurs remarquer qu'arec les dimensions indiquées un petil écarl de ces dimensions n'entraìne pas de variation appréciable dans les résultats des mesures, de sorte que la reproduction des conditions expérimentales indiquées ne présente aucune difficulté.

La méthode qui vient d'itre décrite peut être wilisée pour doser des quantités de radium bien plus faibles que celles qui ont été utilisées pour l'étalonnage. En augmentant le volume du barboteur et la sensibilité du dispositif de mesures, on peut doser ainsi une quantité de radium de l'ordre de $10^{--9} \mathrm{gr}$. contenu dans un volume de liquide qui peut atteindre $50 \mathrm{~cm}^{5}$. Si la dilution de la matière est trop grande et que l'on veuille éviter l'ébullition de grandes masses de liquide, on peut avoir a vantage à précipiter d'abord le radium contenu dans la solution par l'acide sulfurique, à redissoudre le précipité et à doser cnsuite le radium dans cett 3 solution de volume réduit. La précipitation du radium contenu dans une solution en très faible proportion peut être rendue très complète, si la solution contient un pen de baryum, et si la précipitation est répétée deux ou trois fös.

[Immserit recu le 20 mars 1910.$]$ 